$\mathbb{P}$ periodica polytechnica

Chemical Engineering

57/1-2 (2013) 55, 61

doi: 10.3311/PPch.2171

http://periodicapolytechnica.org/ch

Creative Commons Attribution (i)

RESEARCH ARTICLE

\section{Graft copolymerization of acrylic acid onto hydrolyzed potato starch using various initiators}

Suzana Djordjevic / Ljubisa Nikolic / Stana Kovacevic / Milena Miljkovic / Dragan

Djordjevic

Received 2012-12-08, accepted 2013-03-05

\begin{abstract}
This work presents synthesis and characterization of copolymer obtained by grafting of acrylic acid on hydrolyzed potato starch in the presence of various initiators (azobisisobutyronitrile, potassium persulfate and benzoyl peroxide). Starch hydrolysis was made to reduce average molar mass, i.e. to reduce macromolecule size. Grafting of monomer was performed to produce a product that can be used in textile treatment, for example, in yarn sizing. Various initiators were used, primarily, to produce graft polymer on starch and to obtain thinner or thicker packaging of side chains that can have a great influence on the behavior of textile yarns impregnated with these copolymers. Potassium persulfate, as initiator, proved to be very successful in grafting of acrylic acid on hydrolyzed starch, in terms of yield results, graft percentage and efficiency. FTIR spectra of hydrolyzed and grafted starch confirm by their characteristic bands that grafting was successful. Results show that the highest quantity of monomer is found in the sample of hydrolyzed starch grafted in the presence of benzoyl peroxide. Molar masses of hydrolyzate and copolymer show significant differences ranging from $1.83 \times 10^{3}$ to $7.13 \times 10^{6} \mathrm{~g} / \mathrm{mol}$.
\end{abstract}

\section{Keywords}

starch $\cdot$ acrylic acid $\cdot$ grafting $\cdot$ HPLC $\cdot$ molar mass

\section{Suzana Djordjevic}

University of Nis, Faculty of Technology, Leskovac, Serbia

e-mail: szn971@yahoo.com

\section{Ljubisa Nikolic}

University of Nis, Faculty of Technology, Leskovac, Serbia

\section{Stana Kovacevic}

University of Zagreb, Faculty of Textile Technology, Zagreb, Croatia

e-mail: stana.kovacevic@ttf.hr

\section{Milena Miljkovic}

University of Nis, Faculty of Mathematics and Natural Sciences, Nis, Serbia

\section{Dragan Djordjevic}

University of Nis, Faculty of Technology, Leskovac, Serbia

e-mail: drdrag64@yahoo.com

\section{Introduction}

Starch possesses valuable properties like biodegradability, abundant resources, lower environmental contamination, low cost, adhesive properties, ability to make layers, etc. High amounts of starch are used as textile yarn sizing agent and in papermaking. Starch is a polysaccharide consisting of anhydroglucose units (each having three hydroxyl groups) joined by D-glucose bonds. Cyclic structure and many hydroxyl groups make starch material brittle and rigid. Therefore, starch films are not flexible and adhesion to surfaces is never good enough [1]-[3].

No matter whether it is used in textile industry, for example as a sizing agent, or in papermaking, adequate and sufficient adhesion to fibers or paper is considered the most significant characteristic for this kind of applications. For example, in warp sizing process in textile industry, adhesion reduces yarn hairiness by sticking protruding fibers back to yarn body enabling smooth weaving operation. Recently, there is an increasing interest for this and a relevant number of investigation reports appeared [4,5].

Chemical modification offers new possibilities to improve starch inherent shortcomings. Currently, research to improve starch adhesion to fibers is focused to chemical modification. Several techniques are used with the aim to introduce a chemical group or to modify starch molecules in some way to improve adhesion. Among all procedures, graft polymerization of vinyl monomers to starch looks like very fascinating research field having unlimited potentials for improvement of starch properties [6,7].

The objectives of this study are investigation of feasibility and efficiency of creating polymer materials intended for specific end use, e.g. yarn sizing in textile industry. The study involves acid hydrolysis of potato starch to control molecular sizes and then the subsequent graft copolymerization of acrylic acid. Final product designed in this way, hydrolyzed starch grafted with acrylic acid can be used in textile industry for yarn sizing or textile finishing. 


\section{Experimental}

\section{Materials and methods}

In this work, the following chemicals and reagents were used: potato starch (Elton Corporation, Serbia; moisture 17.4\%; ash $0.21 \%$ ), hydrochloric acid (Centrohem, Serbia), ethyl alcohol (Reahem, Serbia), sodium carbonate (LG Hemija, Serbia).

Initiators for grafting process were: Azobisisobutyronitrile (AIBN) [2,2'-azobis(2-methylpropionitrile)] 99\% (SigmaAldrich, USA), potassium persulfate (PP) $\mathrm{K}_{2} \mathrm{~S}_{2} \mathrm{O}_{8}$ (Centrohem, Serbia) and benzoyl peroxide (BP) $\left(\mathrm{C}_{6} \mathrm{H}_{5} \mathrm{CO}\right)_{2} \mathrm{O}_{2}$ (SigmaAldrich, USA).

In grafting process, monomer used was acrylic acid (AA) $\mathrm{C}_{3} \mathrm{H}_{4} \mathrm{O}_{2}$ (Merck, Germany).

\section{Synthesis}

Starch hydrolysis [8,9]: Starch hydrolysis was carried out in aqueous $1 \mathrm{M}$ hydrochloric acid solution at $6{ }^{\circ} \mathrm{C}$ with the addition of starch slurry. After reaction time expired, reaction product was precipitated in $100 \mathrm{~mL}$ ethyl alcohol and neutralized with diluted $1 \%$ sodium carbonate solution, washed with distilled water and finally dried in electric oven at $60^{\circ} \mathrm{C}$ for $3 \mathrm{~h}$. Hydrolysis was carried out with vigorous stirring on magnetic stirrer.

Grafting copolymerization - initiators AIBN, $P P, B P[10]-$ [12]: $10 \mathrm{~g}$ of hydrolyzed starch dissolved in water was heated in water bath at $50{ }^{\circ} \mathrm{C}$ for $15 \mathrm{~min}$. Then, $5 \mathrm{~g}$ of acrylic acid was added and $1 \%$ of initiator (based on starch and monomer mass) was added. Temperature was kept at $50{ }^{\circ} \mathrm{C}$ for $120 \mathrm{~min}$ under reflux with vigorous stirring on magnetic stirrer. Reaction product was precipitated in $100 \mathrm{~mL}$ of ethanol. After filtration, precipitate was washed with $10 \mathrm{~mL}$ of ethanol at ambient temperature and then 3-4 times with ethanol-water solution (80:20) [13]. Finally, the precipitate was dried in electric oven at $60{ }^{\circ} \mathrm{C}$. The product had white colour.

Designations of final products used in text are: HS (hydrolyzed starch), GHS-AIBN (grafted hydrolyzed starch azobisisobutyronitrile), GHS-PP (grafted hydrolyzed starch potassium persulfate) and GHS-BP (grafted hydrolyzed starch - benzoyl peroxide).

Preliminary testing showed the above concentrations and temperature - time conditions of hydrolysis and grafting give the best results.

\section{Characterization methods}

Some grafting parameters was evaluated using following equations [14, 15]:

- Hydrolysis yield [\%]:

$$
\frac{w_{1}}{w_{0}} \cdot 100
$$

- Graft percentage [\%]:

$$
\frac{w_{2}-w_{1}}{w_{1}} \cdot 100
$$

- Graft yield [\%]:

$$
\frac{w_{2}}{w_{1}+w_{3}} \cdot 100
$$

- Graft efficiency [\%]:

$$
\frac{w_{2}-w_{1}}{w_{3}-w_{4}} \cdot 100
$$

- Monomer to polymer conversion [\%]:

$$
\frac{w_{3}-w_{4}}{w_{3}} \cdot 100
$$

Where $w_{1}$ is mass of hydrolyzed starch $(\mathrm{g}), w_{0}$ is mass of native starch $(\mathrm{g}), w_{2}$ is mass of grafted starch $(\mathrm{g}), w_{3}$ is mass of monomer used $(\mathrm{g})$ and $w_{4}$ is mass of residual monomer $(\mathrm{g})$.

Determination of average copolymer molecular weights and distribution of molecular weights

Average molecular mass and molecular mass distribution was determined using GPC Agilnet 1100 Series gel permeation chromatograph with differential refractometer (RID detector) 1200 Series used as detector. Zorbax PSM 300, $250 \cdot 6.2 \mathrm{~mm}, 5 \mu \mathrm{m}$ column having nominal molar mass range of $3 \times 10^{3}-3 \times 10^{5}$ $\mathrm{g} / \mathrm{mol}$ was used. As eluent, redistilled water with flow rate of 1 $\mathrm{mL} / \mathrm{min}$ was used. The column was conditioned at $25^{\circ} \mathrm{C}$ and injected volume of sample solution was $20 \mu \mathrm{L}$. The copolymer solutions in redistilled water with concentration of $4.5 \mathrm{mg} / \mathrm{mL}$, filtered before weighing through $0.45 \mu \mathrm{m}$ filter, were used for analysis.

In order to make calibration curve to quantitatively process gel-chromatograms of tested starch copolymers, calibration of Zorbax PSM-300 gel column was carried out using narrow molar mass distribution dextran samples (Pharmacia). Dextran standards of known molar mass and the same concentrations as those of the samples were used in the same way as the samples.

Based on chromatography parameters obtained, automatic analysis with GPC software and linear curve fitting was used for optimization and selection of calibration curve.

\section{Determination of residual monomer in copolymer}

Using liquid chromatography method under high pressure (High Performance Liquid Chromatography, HPLC), residual quantities of the unreacted monomer were determined in the product material. For analysis, about $0.2 \mathrm{~g}$ of powdered copolymer sample was taken. The sample was extracted with $10 \mathrm{~mL}$ of methanol at ambient temperature for $48 \mathrm{~h}$ with intermittent stirring on magnetic stirrer. Extracts were filtered through $0.45 \mu \mathrm{m}$ filter and used for HPLC analysis. Recordings were performed on HPLC Agilent 1100 Series apparatus with DAD 1200 Series diode-array detector. Detection wavelength was $205 \mathrm{~nm}$. The column used was ZORBAX Eclipse XDB-C18, $4.6 \times 250 \mathrm{~mm}$, $5 \mu \mathrm{m}$. Eluent was methanol with flow rate of $1 \mathrm{~mL} / \mathrm{min}$, column was conditioned at $25^{\circ} \mathrm{C}$ and injection volume $20 \mu \mathrm{L}$.

As calibration standard, the samples of acrylic acid were used in concentrations of $1-0.005 \mathrm{mg} / \mathrm{L}$. Standard monomers were 
prepared by weighing individual samples in volumetric flasks and diluting them with mobile phase i.e. methanol.

\section{Results}

Starch hydrolysis was carried out to reduce its molecular weight. Acrylic acid is then grafted on shorter starch molecules forming side branches on the main chain, resulting in a product that can be used more successfully in textile processing. Variation of initiators was intended, primarily, to create graft monomer on starch, more efficiently, and to produce various thinner or thicker, shorter or longer side chains. The variations have an impact on final product tailoring and its behavior in contact with textile.

During copolymerization with initiators AIBN, BP and PP, the formed grafted branches bind with starch backbone via oxygen because graft copolymerization is initiated by expelling active hydrogen atom from starch hydroxyl, Figure 1] [2, 16].

This scheme is based on a fact that both the C1-C2 (end groups) and C2-C3 are predominant sites for the initiation of graft copolymerization [17]. Most commonly, graft polymerization is brought about by free radical addition polymerization. Even though a large number of reports $[18]-[24]$ on graft copolymerization has been published, there is not a comprehensive understanding of the grafting process yet.

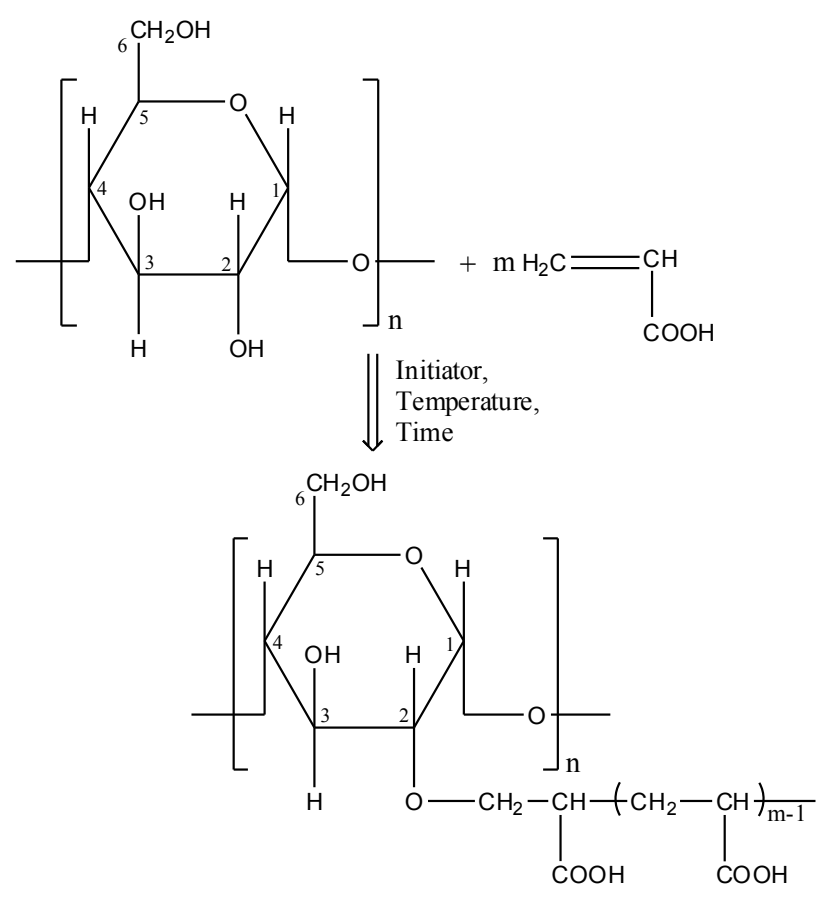

Fig. 1. Scheme of acrylic acid grafting on hydrolyzed starch

Table 1 shows the efficiency parameters of hydrolysis and monomer grafting on starch depending on initiator type.

As an initiator, PP proved to be very successful in acrylic acid grafting on starch, in terms of yield results, grafting percentage and efficiency. Grafting in the presence of AIBN and BP initiators has slightly better monomer-to-polymer conversion values (difference is on first decimal), which, considering other pa- rameters in Table 1 , indicate that higher conversion can produce unproductive reaction - producing more homopolymer and less graft polymer.

Increasing the grafting efficiency is an effective way of improving the properties of starch graft copolymers and reducing their preparation costs [18, 19]. However, the graft copolymerization of starch with vinyl monomers is always accompanied by the homopolymerization of vinyl monomers as reactants. The homopolymerization is a side reaction of the copolymerization. It can be imagined that, through the introduction of polymerizable double bonds into starch molecules before graft copolymerization, the double bonds can be incorporated into copolymeric chains when they meet propagating activated chains. If a propagating activated chain that initiates homopolymerization meets with the double bonds, the original homopolymerization will be converted into copolymerization.

Therefore, some portion of the homopolymers will become graft copolymers, and the grafting efficiency can be enhanced. Consequently, the grafting ratio will be correspondingly enhanced with the increase in the efficiency. It has been elucidated that the homopolymers are inferior to the copolymers in serviceability. As a result, increasing the grafting efficiency enhances the serviceability of the products. On the other hand, if the grafting ratio is similar, the enhancement of the grafting efficiency decreases the required amounts of the monomers, and this reduces the production cost [21,22].

The rise in graft efficiency and grafting percentage (GHS-PP sample) may be attributed to the formation of increasing number of free radicals on the starch backbone at which the monomer molecules can be grafted.

The decline in graft efficiency and grafting percentage at other samples (GHS-BP and GHS-AIBN) may be rationalized as follows:

(1) non-availability of sites on starch molecules at which initiators can react to generate more free radicals;

(2) the initiation of homopolymerization by unutilized initiators.

The decrease in grafting yield at GHS-BP sample may be owing to the wastage of AA molecules in the formation of large amounts of homopolymer. Formed homopolymer lumps then progressively hinder the rate of diffusion of monomer molecules to the starch macroradicals, resulting in the gradual decrease in graft efficiency and grafting percentage. Needless to say, the starch macroradicals are comparatively immobile and, hence, the grafting efficiency will exclusively depend on the diffusion of monomer molecules in their vicinity.

The levelling off of graft efficiency is, perhaps, a direct consequence of depletion of monomer available for grafting.

Figure 2 shows parallel FTIR spectra of hydrolyzed starch and starch grafted with various initiators. In FTIR spectrum of hydrolyzed starch a broad absorption band at $3395 \mathrm{~cm}^{-1}$ for $\mathrm{O}-\mathrm{H}$ stretching vibrations and a smaller absorption band at $2925 \mathrm{~cm}^{-1}$ attributed to $\mathrm{C}-\mathrm{H}$ stretching vibration are observed. Wave num- 
Tab. 1. Some process parameters of starch hydrolysis and grafting

\begin{tabular}{lccccc}
\hline Sample & $\begin{array}{c}\text { Hydrolysis yield } \\
\%\end{array}$ & $\begin{array}{c}\text { Grafting yield } \\
\%\end{array}$ & $\begin{array}{c}\text { Grafting percentage } \\
\%\end{array}$ & $\begin{array}{c}\text { Graft efficiency } \\
\%\end{array}$ & $\begin{array}{c}\text { Monomer to polymer } \\
\text { conversion, } \%\end{array}$ \\
\hline GHS-AIBN & - & 86.11 & 26.8 & 57.36 & 98.88 \\
GHS-BP & - & 84.21 & 24.0 & 51.70 & 98.25 \\
GHS-PP & - & 88.15 & 29.8 & 63.56 & 99.23 \\
HS & 90.80 & - & - & - & - \\
\hline
\end{tabular}

bers at 1153,1078 and $1020 \mathrm{~cm}^{-1}$ describe $\mathrm{C}-\mathrm{O}-\mathrm{C}$ stretching (triplet for starch) and a band at $1638 \mathrm{~cm}^{-1}$ corresponds to water molecule [25].

In the case of hydrolyzed starch grafted with acrylic acid, characteristic bands, assigned to polyacrylic acid, appeared. Carboxyl acid group was detected by absorption of carbonyl $\mathrm{C}=\mathrm{O}$ group at about $1730 \mathrm{~cm}^{-1}(\mathrm{C}=\mathrm{O}$ stretching $)$. With carboxylate anion, two $v(\mathrm{C}=\mathrm{O})$ maximums appear, one at about $1560 \mathrm{~cm}^{-1} v_{a s}(\mathrm{C}=\mathrm{O})$ and the other at about $1408 \mathrm{~cm}^{-1} v_{s}(\mathrm{C}=\mathrm{O})$. Broad band in the region of $3400 \mathrm{~cm}^{-1}$ indicates the presence of $\mathrm{OH}$ groups, bands below $3000 \mathrm{~cm}^{-1}$ indicate $v(\mathrm{C}-\mathrm{H})$ valent vibrations of saturated carbon atoms, $\delta(\mathrm{C}-\mathrm{H})$ absorption bands of methyl groups appear at about $1375 \mathrm{~cm}^{-1}$, and of methylene groups in the region of $1450-1370 \mathrm{~cm}^{-1}$ [1,2].

Concentration of AA monomer is calculated from peak area on HPLC chromatogram using linear equation $(y=a x+b)$, obtained calibrating relationship between absorption peak area and monomer concentration in eluted sample. AA monomer retention time is $2.32 \mathrm{~min}$. From Figure 3 it can be observed that linear relationship ranges to AA concentration of $0.1 \mathrm{mg} / \mathrm{mL}$, i.e. up to peak area of $4660 \mathrm{mAU} \cdot \mathrm{s}$. For linear part of relationship between peak area and AA monomer concentration the following equation is applicable $y=42922.12 \cdot x+0$, where: $x$ $(\mathrm{mg} / \mathrm{mL})$ is acrylic acid concentration; $y(\mathrm{mAU} \cdot \mathrm{s})$ is peak area with retention time $R_{t}=2.32 \mathrm{~min}$. Since zero is the origin of the calibration curve, there is no ordinate intercept.

High linearity is confirmed by statistic parameters, coefficient of determination $\mathrm{R}^{2}$ of 0.986 .

The quantities of residual monomer in copolymer samples after acrylic acid grafting on hydrolyzed starch are: $4.17 \mathrm{mg} / \mathrm{g}$ (GHS-AIBN), $6.66 \mathrm{mg} / \mathrm{g}$ (GHS-BP) and $2.80 \mathrm{mg} / \mathrm{g}$ (GHS-PP).

The highest amount of monomer was found in GHS-BP sample, i.e. using benzoyl peroxide initiator in monomer grafting on starch.

Figure 4 shows a HPLC chromatogram (RID signal) of aqueous extract of synthesized starch copolymer sample depending on type of initiator used, indicating that, with given conditions and used initiators, the corresponding response of copolymer chromatograph signal appeared. It can be observed that grafting in the presence of AIBN and BP initiators give starch-AA copolymer having highest intensity in chromatogram, the higher area and narrower distribution of macromolecules in appropriate range of molecular weights. Chromatogram details for copolymer samples are given in Table 2. On the characteristic reten- tion time, hydrolyzed starch has the largest area with somewhat lower intensity and a broad base peak.

In Table 3 shown are details of various molar mass types of copolymer samples and polydispersity index calculated from distribution curve. Higher values for $\mathrm{Mn}, \mathrm{Mw}$ and $\mathrm{Mz}$ molar masses are result of higher fraction of higher molar masses.

Tab. 2. Some chromatogram parameters of copolymer samples

\begin{tabular}{lccr}
\hline Sample & $\begin{array}{c}\text { Retention time } \\
\text { min }\end{array}$ & $\begin{array}{c}\text { Peak area } \\
\mathrm{mAU} \cdot \mathrm{s}\end{array}$ & $\begin{array}{c}\text { Peak height } \\
\mathrm{mAU}\end{array}$ \\
\hline GHS-AIBN & 5.219 & 464623.0 & 21420.0 \\
GHS-BP & 5.180 & 524846.4 & 20374.0 \\
GHS-PP & 5.174 & 253251.5 & 9413.4 \\
HS & 5.255 & 486118.5 & 18742.6 \\
\hline
\end{tabular}

Results are consistent with known facts that natural polymer molecules, for example polysaccharides and practically all synthetic polymers have different sizes; i.e. these polymers are polydisperse. In our case, the sample of hydrolyzed starch grafted with acrylic acid in the presence of benzoyl peroxide initiator show the highest uniformity. Of course, various size molecules having different fractions can be found in material samples.

As with all tested samples $M_{w}>M_{n}$ it could be concluded that they are polydisperse systems. The higher the difference between $M_{w}$ and $M_{n}$, the more polydisperse is the system. It is known that all synthetic polymers are polydisperse with the following order: $M_{z}>M_{w}>M_{n}$.

Increased polydispersity index (sample GHS-PP) could mean the presence of higher content of lower molecular chains having consequently higher mobility.

Higher chain lengths, respectively higher molar masses, provide higher number of interactions and higher interlacement probability of flexible chains in material resulting in higher strength and elasticity and other properties as well. Therefore, to optimize the properties, compromise values of these parameters are required. Variation of polydispersity index gives additional freedom in designing polymer materials. Higher dispersibility provides, for example, better processing. Explanation of this phenomenon could be found in the fact that shorter chains are more mobile in melt and serve as 'lubricants' during movement of long chains and their aggregates.

If we look at the connection between the grafting parameters and molecular weight of copolymers it can be said that the molecular weights seem to grow almost linearly with conver- 


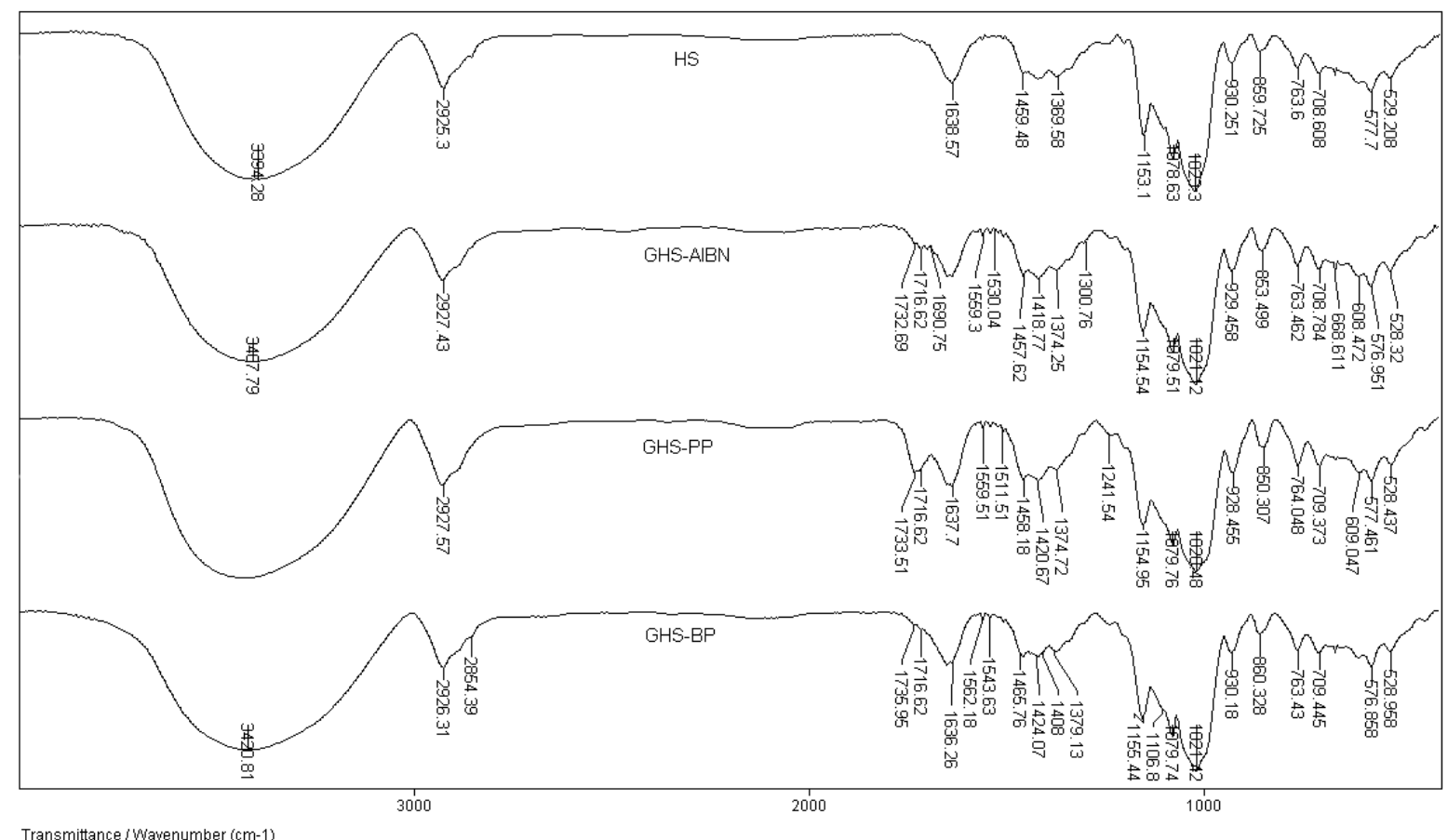

Fig. 2. FTIR spectra of hydrolyzed and acrylic acid grafted starch

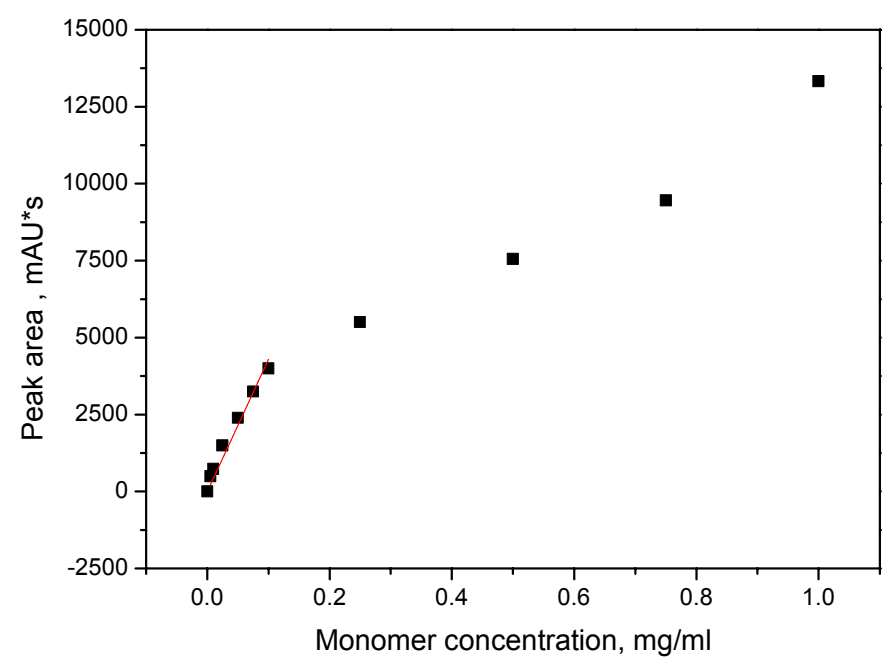

a.

Fig. 3. Calibration curve for determination of monomer concentration in extracted copolymer samples using HPLC method (a. - full concentration range; b. - linear part)

sion, at least with the limited amount of data points available. However, also the intermolecular termination has an effect on the molecular weight of the samples, so no exact conclusions can be drawn from this dependency.

When we recall the data in Table 3 that the molecular weights of HS sample and grafted starches are similar, i.e. there are small differences, it seems certain that a growing chain radical in a micelle reacts with the starch surface via a yet unidentified route. Another possibility is that a growing chain radical in a micelle has to react with starch. It may be visualized that a growing chain radical in a micelle is adsorbed on the starch surface and attached to an oxy radical at the starch surface [26].

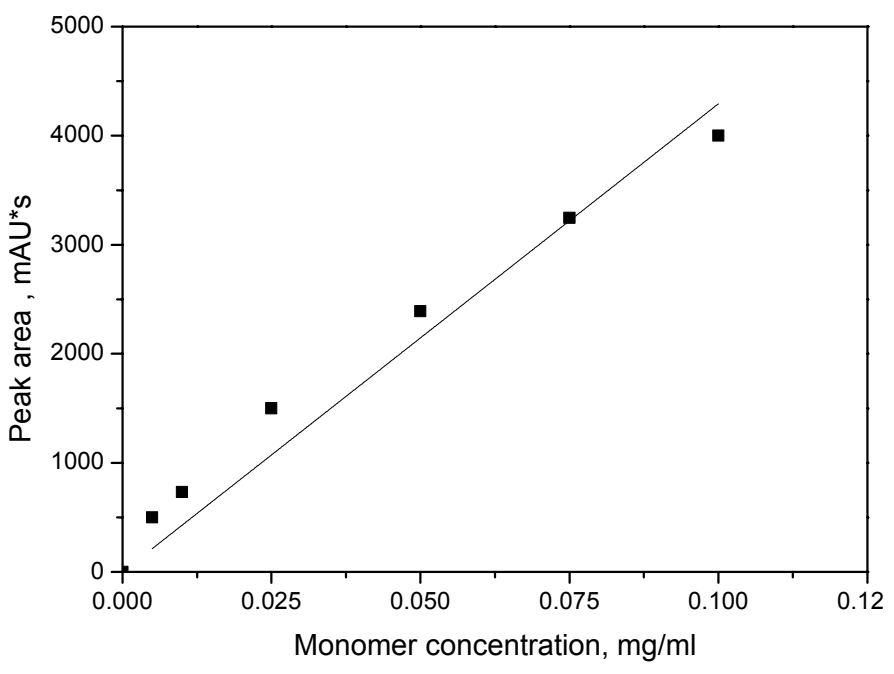

b.

\section{Conclusions}

Upon hydrolysis, some starch granules are fractured to lowmolecular-weight fragments, which are composed of amyloserich and/or amylopectin-rich domains. The properties of the HS gel depend both on the continuous phase (solubilized amylose) and the dispersed phase (the granule residues), as well as on the interaction of these two phases. The rate of recombination of the more hydrolyzed chains is higher than that of the less hydrolyzed ones.

With these points in mind, it is clear that the interaction of AA with the hydrolyzed starch depends largely upon the molecular size and nature of the hydrolyzed starch by virtue of their 


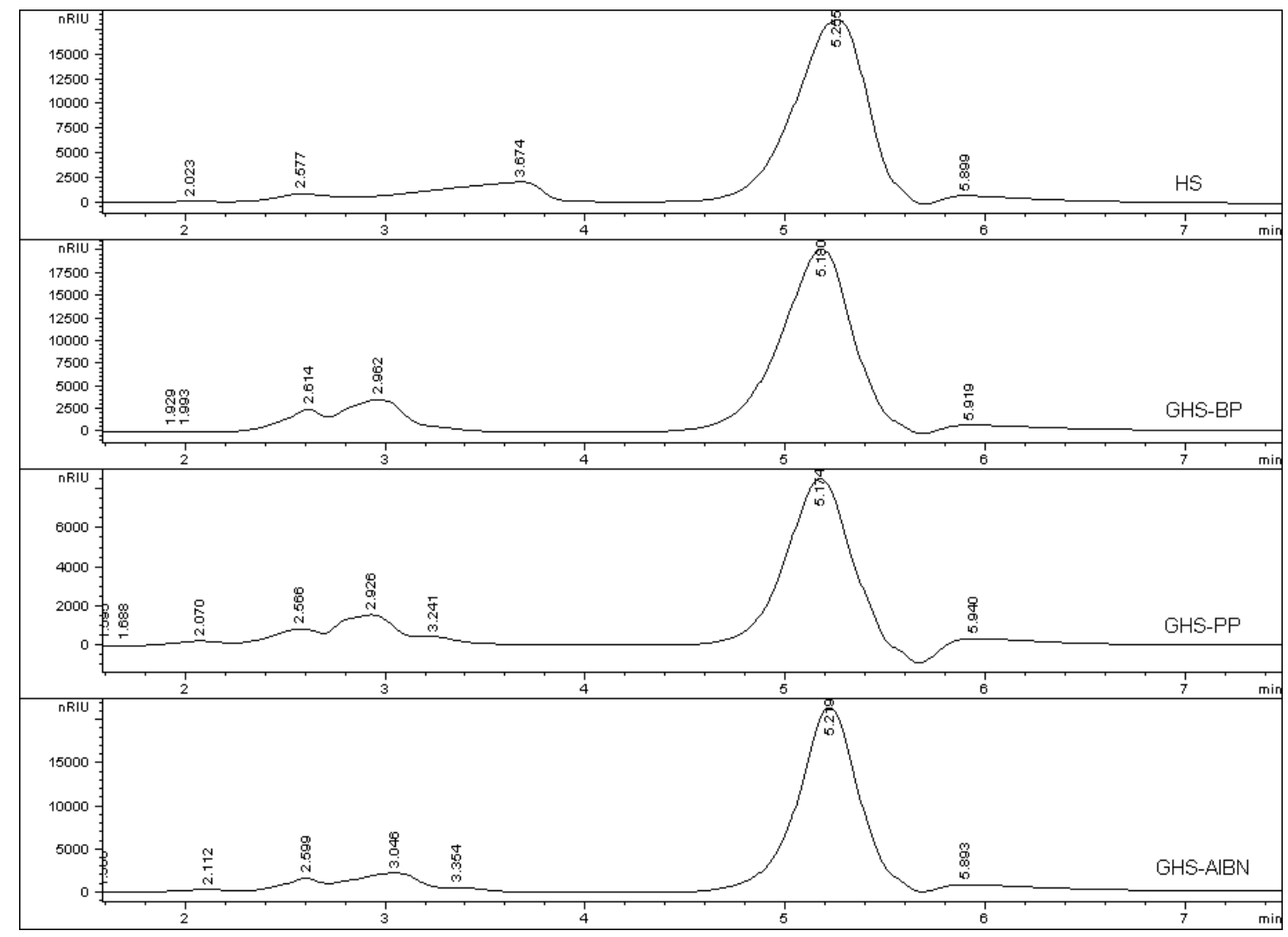

Fig. 4. HPLC chromatogram of starch-AA copolymer.

Tab. 3. Average molar masses $(\mathrm{g} / \mathrm{mol})$ and polydispersity index and polymer in relation to initiator from GPC curves

\begin{tabular}{|c|c|c|c|c|c|c|c|c|}
\hline \multirow{3}{*}{ Sample } & \multicolumn{8}{|c|}{ Elution volume, $\mathrm{mL}$} \\
\hline & \multicolumn{4}{|c|}{$1.8-4$} & \multicolumn{4}{|c|}{$4.3-6.5$} \\
\hline & $\mathrm{Mn}^{\mathrm{b}}$ & $M w^{c}$ & $\mathbf{M z}^{\mathrm{d}}$ & $P D^{e}$ & Mn & Mw & Mz & PD \\
\hline GHS-AIBN & $1.06 \times 10^{6}$ & $1.76 \times 10^{6}$ & $2.67 \times 10^{6}$ & 1.65 & $3.82 \times 10^{3}$ & $2.94 \times 10^{3}$ & $3.82 \times 10^{3}$ & 1.62 \\
\hline GMHS-BP & $1.42 \times 10^{6}$ & $2.07 \times 10^{6}$ & $2.95 \times 10^{6}$ & 1.45 & $3.82 \times 10^{3}$ & $3.66 \times 10^{3}$ & $5.02 \times 10^{3}$ & 1.54 \\
\hline GHS-PP & $1.34 \times 10^{6}$ & $2.89 \times 10^{6}$ & $7.13 \times 10^{6}$ & 2.15 & $3.82 \times 10^{3}$ & $3.36 \times 10^{3}$ & $4.22 \times 10^{3}$ & 1.63 \\
\hline HS & $3.29 \times 10^{6}$ & $9.58 \times 10^{6}$ & $2.63 \times 10^{6}$ & 2.91 & $3.82 \times 10^{3}$ & $3.00 \times 10^{3}$ & $3.97 \times 10^{3}$ & 1.46 \\
\hline
\end{tabular}

${ }^{\mathrm{b}}$ Number average molar mass; ${ }^{\mathrm{C}}$ Weight average molar mass;

${ }^{\mathrm{d}} \mathrm{Z}$ Average molar mass; ${ }^{\mathrm{e}}$ Polydispersity index, $D=M w / M n$

direct impact on the initiation system; the swellability of the starch; diffusion and adsorption of AA; molecular collisions; reactions involving primary free radicals, starch radicals and growing poly(AA) chain radicals; etc.

Grafted product shows different properties depending on used initiator type. Initiator potassium persulfate found to be very successful in acrylic acid grafting on hydrolyzed starch, in terms of yield, graft percentage and efficiency.

FTIR spectra of hydrolyzed and grafted starch confirm by characteristic absorption bands that grafting of monomer was successful.

Quantity of residual monomer in polymer, determined chromatographically, was the highest in the sample of hydrolyzed starch grafted with benzoyl peroxide.
Molar masses of tested hydrolyzate and copolymer samples show a difference and higher variation in the range of $1.81 \times 10^{3}$ to $7.13 \times 10^{6} \mathrm{~g} / \mathrm{mol}$.

Higher values of polydispresity index could mean the presence of higher content of lower molar mass chains with consequently higher mobility required for further use, e.g. in yarn sizing process or textile finishing.

\section{References}

1 Biswas A, Willet JL, Gordon SH, Finkenstadt VL, Cheng HN, Complexation and blending of starch, poly(acrylic acid), and poly( $N$-vinyl pyrrolidone), Carbohyd. Polym., 65, (2006), 397-403.

2 Zhu Z, Li M, Jin E., Effect of an Allyl Pretreatment of Starch on the Grafting Efficiency and Properties of Allyl Sta(rch-g-Poly(acrylic acid), J. Appl. Polym. Sci., 112, (2009), 2822-2829. 
3 Mostafa KM, El-Sanabary AA, Harnessing of Novel Tailored Modified Pregelled Starch-Derived Products in Sizing of Cotton Textiles, Adv. Polym. Tech., 31, (2012), 52-62.

4 Li M, Zhu Z, Pan X, Effects of starch acryloylation on the grafting efficiency, adhesion, and film properties of acryloylated starch-g-poly(acrylic acid) for warp sizing, Starch/Starke, 63, (2011), 683-691.

5 Huacai G, Wan P, Dengke L, Graft copolymerization of chitosan with acrylic acid under microwave irradiation and its water absorbency, Carbohyd. Polym., 66, (2006), 372-378.

6 Da-Wei H, Jian-Kun W, Hai-Rong L, Ming-Ming X, The Graft Copolymerization of Corn Starch by Microwave Irradiation with Rheological Phase Reaction, J. Appl. Polym. Sci., 121, (2011), 1481-1487.

7 Lee JS, Kumar RN, Rozman HD, Azemi BMN, Pasting, swelling and solubility properties of UV initiated starch-graft-poly(AA), Food Chem., 91, (2005), 203-211.

8 Kiatkamjornwonga S, Chomsaksakul W, Sonsukc M, Radiation modification of water absorption of cassava starch by acrylic acid/acrylamide, Radiat. Phys. Chem., 59, (2000), 413-427.

9 Biswas A, Willet JL, Gordon SH, Finkenstadt VL, Cheng HN, Complexation and blending of starch, poly(acrylic acid), and poly( $N$-vinyl pyrrolidone), Carbohyd. Polym., 65, (2006), 397-403.

10 Carvalho AJF, Curvelo AAS, Gandini A, Surface chemical modification of thermoplastic starch: reactions with isocyanates, epoxy functions and stearoyl chloride, Ind. Crop. Prod., 21, (2005), 331-336.

11 Kaewtatip K, Tanrattanakul V, Preparation of cassava starch grafted with polystyrene by suspension polymerization, Carbohyd. Polym., 73, (2008), 647-655.

12 Isklan N, Kursun F, Inal M, Graft copolymerization of itaconic acid onto sodium alginate using benzoyl peroxide, Carbohyd. Polym., 79, (2010), 665672.

13 Mostafa KM, Synthesis of poly(acrylamide)-starch and hydrolyzed starch graft copolymers as a size base material for cotton textiles, Polym. Degrad. Stabil., 55, (1997), 125-130.

14 Meshram MW, Patil VV, Mhaske ST, Thorat B. N., Graft copolymers of starch and its application in textiles, Carbohyd. Polym., 75, (2009), 71-78.
15 Song $\mathbf{H}$, Synthesis and application of cationic starch graft poly mer by using the complec initiation system, Carbohyd. Polym., 82, (2010), 768-771.

16 Witono JR, Noordergraaf IW, Heeres HJ, Janssen LPBM, Graft copolymerization of acrylic acid to cassava starch - Evaluation of the influences of process parameters by an experimental design method, Carbohyd. Polym., 90, (2012), 1522-1529.

17 Doba T, Rodehed C, Ranby B, Mechanism of graft copolymerization onto polysaccharides initiated by metal ion oxidation reactions of model compounds for starch and cellulose, Macromolecules, 17, (1984), 3181-3187.

18 Aravindakshan P, Kumar VG, Effect of grafting with acrylic monomers on the viscosity, gelatinization temperature, and granule swelling characteristics of starch, J. Appl. Polym. Sci., 84, (2002), 528-534.

19 Athawale VD, Rathi SC, Graft polymerization: Starch as a model substrate, J. Macromol. Sci. R. M. C., C39, (1999), 445-480.

20 Bayazeed A, Elzairy MR, Hebeish A, Synthesis and application of new thickeners Part I: Preparation of poly(acrylic acid) - starch graft copolymer, Starch/Stärke, 41, (1989), 233-236.

21 Fakhru'l-Razi A, Qudsieh IYM, Wan Yunus WMZ, Ahmad MB, Graft copolymerization of methyl methacrylate onto sago starch using ceric ammonium nitrate and potassium persulfate as redox initiator systems, J. Appl. Polym. Sci., 82, (2001), 1375-1381.

22 Fares MM, El-faqeeh AS, Osman ME, Graft copolymerization onto starch. I. Synthesis and optimization of starch grafted with $\mathrm{N}$-tert-butylacrylamide copolymer and its hydrogels, J. Polym. Res., 10, (2003), 119-125.

23 Lutfor MR, Sidik S, Haron J, Rahman MZA, Ahmad M, Modification of sago starch by graft copolymerization: Effect of reaction conditions on grafting parameters, Int. J. Polym. Mater., 52, (2003), 189-201.

24 Sangramsingh NM, Patra BN, Singh BC, Patra CM, Graft copolymerization of methyl methacrylate onto starch using a Ce(IV)-glucose initiator system, J. Appl. Polym. Sci., 91, (2004), 981-990.

25 Zheng Y, Hua S, Wang A, Adsorption behavior of $\mathrm{Cu}^{2+}$ from aqueous solutions onto starch-g-poly (acrylic acid)/sodium humate hydrogels, Desalination, 263, (2010), 170-175.

26 Chang G. C., Kiho L., Preparation of starch-g-polystyrene copolymer by emulsion polymerization, Carbohyd. Polym., 48, (2002), 125-130. 